\title{
Blue Mussel Protein Concentrate Versus Prime Fish Meal Protein as a Dietary Attractant for Turbot (Psetta maxima L.) Given Rapeseed Protein- based Diets
}

\author{
Nagel $F^{1,4}$, Appel $T^{2}$, Rohde $C^{1}$, Kroeckel $S^{1,3}$ and Schulz $C^{1,4 *}$ \\ ${ }^{1}$ Gesellschaft für Marine Aquakultur $\mathrm{mbH}$, Germany, \\ ${ }^{2}$ Institute of Zoology, Christian-Albrechts-Universität zu Kiel, Germany \\ 3Institute of Animal Nutrition and Physiology, Christian-Albrechts-Universität zu Kiel, Germany \\ ${ }^{4}$ Institute of Animal Breeding and Husbandry, Christian-Albrechts-Universität zu Kiel, Germany
}

\begin{abstract}
A feeding experiment was conducted to test the attractant potential of a processed protein concentrate of the blue mussel (BMPC) as replacer for fish meal (FM) protein in rapeseed protein concentrate (RPC)-based diets for turbot. Triplicate fish groups received isonitrogenous and isoenergetic diets with a $50 \%$ or $75 \%$ replacement of $\mathrm{FM}$ protein by a RPC (RPC 50, RPC 75), and further FM protein substitution with $0,2,4$ or $8 \%$ of BMPC. The application of RPC 50/0 provided no significant impact on fish performance compared to FM-reference diet, while daily feed intake (DFI), specific growth rate (SGR) and feed conversion were significantly impaired when fish fed with RPC $75 / 0(P<0.05)$. Incorporation of BMPC failed to significantly improve DFI, SGR and FCR in RPC 50 and RPC 75 treatments $(P>0.05)$. Protein efficiency ratio and protein productive values remained unaffected among all treatments $(P>0.05)$. Excepting crude ash content, no changes in crude protein, crude lipid, dry matter and energy content were obtained. Hepatosomatic index tended to increased $(P>0.05)$ in accordance with slightly hypertrophic hepatocytes of fish fed diets with a BMPC incorporation of $40-80 \mathrm{~g} \mathrm{~kg}^{-1}$. Neither inflammatory nor degenerative alterations were detected in the intestine. In summary, we demonstrated that BMPC failed to stimulate the feed intake of turbot when dietary FM protein was substituted consecutively, but it was found to maintain performance level of turbot within the test diet groups. This indicates nutritional properties of BMPC comparable to prime FM protein, functional to further reduce the FM protein content in aquafeeds for carnivorous fish.
\end{abstract}

Keywords: Stimulant; Palatability; Feed intake; Specific growth rate; Nutrient utilization; Mytilus edulis

\section{Introduction}

Global supply of fishmeal (FM) and fish oil is unlikely to increase beyond current levels due to limited natural resources. In addition, the increasing concern of economic and ecological sustainability are creating a paramount pressure on the aquaculture industry to reduce levels of FM and fish oil in aqua feeds [1] and to seek for alternative sources of protein. It is already apparent that plant protein sources play an important role, especially in the efforts to move the formulation of fish feed "down the food chain". However, the consecutive replacement of FM by plant proteins is still challenging in nutrition of carnivorous fish, such as turbot (Psetta maxima), common sole (Solea solea) or Atlantic halibut (Hippoglossus hippoglossus). Complete FM replacement by plant proteins has been achieved in different species [2-5], but failed to keep fish performance unaffected [6-10]. Diet formulations with increasing levels of plant proteins such as rapeseed meal resulted in significant alterations in digestible nutrient content and levels of antinutritional factors (ANFs) [11].

Commercially, these in turn may affect palatability, feed intake and nutrient utilization as well as growth of carnivorous fish, $[1,11,12]$. Purified rapeseed products are generally characterised by high protein content and a balanced amino acid profile [12]. Furthermore, technical processing have been found to reduce the content of substantial ANFs, inluding glucosinolates, phytic acid, sinapinic acid and tannins. Purification protocols strengthen their application as feed ingredient for some species $[4,5]$, while maintaining restrictions for other feeds and species [9,13-16]. Juvenile turbot showed reduced feed intake and growth performance when FM protein was replaced by $30 \%$ to $75 \%$ (corresponding to $<300 \mathrm{~g} \mathrm{FM} \mathrm{kg}^{-1}$ diet) with rapeseed products
[10,17-19], suggesting rapeseed-derived palatability and digestibility impairments of the diets.

A plurality of studies have attempted to stimulate feed intake and improve palatability of aquafeeds using attractants of various origins including chemical mixtures, natural compounds and tissue extracts [20-25]. Among the resources of marine origin, blue mussels (Mytilus edulis) are indicted by sufficient availability. Their farming is relatively simple and has expanded in several regions [26]. Since mussels smaller than $5 \mathrm{~cm}$ are of minor interest for human nutrition they are sorted out of harvested biomass [27]. Huge production volumes, high protein content and an amino acid pattern similar to fish meal have predestined blue mussels (or derived meals/extracts) for aquafeeds [27,28]. The amino acids glycine and alanine as well as the non-amino acid betaine have been identified as gustatory stimulants and have been detected in high amounts in muscle tissue extracts [27,29]. Mussel meat (either freshly or as freeze-dried meal) has been successfully applied as protein source in diets for rainbow trout (Oncorhynchus mykiss) [27,30],

*Corresponding author: Professor Carsten Schulz, Institute of Animal Breeding and Husbandry, Christian-Albrechts-Universität zu Kiel, Institut für Tierzucht und Tierhaltung, Olshausenstr. 40, 24098 Kiel, Germany, Tel: ++ 49(0)431-8805388; Fax: ++ 49(0)431-8802588; E-mail: cschulz@tierzucht.uni-kiel.de

Received December 22, 2016; Accepted January 19, 2017; Published January 21, 2017

Citation: Nagel F, Appel T, Rohde C, Kroeckel S, Schulz C (2017) Blue Musse Protein Concentrate Versus Prime Fish Meal Protein as a Dietary Attractant for Turbot (Psetta maxima L.) Given Rapeseed Protein-based Diets. J Aquac Res Development S2: 012. doi:10.4172/2155-9546.S2-012

Copyright: ( 2017 Nagel F, et al. This is an open-access article distributed under the terms of the Creative Commons Attribution License, which permits unrestricted use, distribution, and reproduction in any medium, provided the original author and source are credited. 
Citation: Nagel F, Appel T, Rohde C, Kroeckel S, Schulz C (2017) Blue Mussel Protein Concentrate Versus Prime Fish Meal Protein as a Dietary Attractant for Turbot (Psetta maxima L.) Given Rapeseed Protein-based Diets. J Aquac Res Development S2: 012. doi:10.4172/2155-9546. S2-012

Page 2 of 7

\begin{tabular}{|c|c|c|c|}
\hline Variables & FM & RPC & BMPC \\
\hline \multicolumn{4}{|c|}{ Nutrient composition } \\
\hline Dry matter $\left(\mathrm{g} \mathrm{kg}^{-1}\right)$ & 934 & 944 & 893 \\
\hline Crude protein $\left(\mathrm{g} \mathrm{kg}^{-1} \mathrm{DM}\right)$ & 706 & 691 & 728 \\
\hline Crude fat $\left(\mathrm{g} \mathrm{kg}^{-1} \mathrm{DM}\right)$ & 122 & 16 & 30 \\
\hline $\mathrm{NfE}+$ crude fibre $\left(\mathrm{g} \mathrm{kg}^{-1} \mathrm{DM}\right)$ & 0 & 195 & 141 \\
\hline Crude ash $\left(\mathrm{g} \mathrm{kg}^{-1} \mathrm{DM}\right)$ & 172 & 98 & 101 \\
\hline Gross energy (MJ kg $\left.{ }^{-1} \mathrm{DM}\right)$ & 21.2 & 20.6 & 21.3 \\
\hline \multicolumn{4}{|c|}{ Essential amino acids (g $\left.100 \mathrm{~g}^{-1} \mathrm{CP}\right)$} \\
\hline Arginine & 6.6 & 7.6 & 7.2 \\
\hline Histidine & 2.2 & 3.0 & 2.1 \\
\hline Isoleucine & 4.1 & 4.4 & 4.2 \\
\hline Leucine & 6.8 & 8.0 & 6.7 \\
\hline Lysine & 6.8 & 5.8 & 7.0 \\
\hline Methionine + Cystine & 3.5 & 4.3 & 3.2 \\
\hline Phenylalanine & 3.7 & 4.5 & 3.7 \\
\hline Threonine & 4.1 & 4.4 & 4.8 \\
\hline Valine & 4.8 & 5.3 & 6.7 \\
\hline \multicolumn{4}{|c|}{ Anti-nutritional factors } \\
\hline Glucosinolates $\left(\mu \mathrm{mol} \mathrm{g}{ }^{-1}\right)$ & - & $<0.1$ & - \\
\hline Sinapinic acid and sinapinic acid ester $\left(\mathrm{g} \mathrm{kg}^{-1}\right)$ & - & 0.024 & - \\
\hline Phytic acid $\left(\mathrm{g} \mathrm{kg}^{-1}\right)$ & - & 22.6 & - \\
\hline Tannins $\left(\mathrm{g} 100 \mathrm{~g}^{-1}\right)$ & - & $<0.1$ & - \\
\hline Mustard seed oil $\left(\mathrm{g} \mathrm{kg}^{-1}\right)$ & - & $<0.1$ & \\
\hline
\end{tabular}

Table 1: Nutrient composition, amino acid profile and antinutritional factors (glucosinolates, sinapinic acid, phytic acid, tannins and mustard seed oil) of fish meal (FM), rapeseed protein concentrate (RPC) and blue mussel protein concentrate (BMPC).

red sea bream (Pagrus major) [31], Japanese flounder (Paralichthys olivaceus) $[32,33]$ and common sole (Solea solea) $[20,34]$. Furthermore, mussel meat or solvent extracts have been found to be efficient feed attractants in diets for common sole [29] or Japanese flounder $[35,36]$ and tiger puffer (Takifugu rubripes) $[28,33]$. For turbot, it was demonstrated recently, that low inclusion levels of crude blue mussel meal (substituted with wheat gluten) were found to diminish rapeseedderived palatability impairments by increasing daily feed intake significantly [37]. However, studies utilizing technologically processed blue mussel protein concentrate (BMPC) as attractant in alternative diets have been hardly investigated. Therefore, the aim of the present study was to evaluate the attractant potential of a BMPC substituting high quality FM protein in a rapeseed protein concentrate-based diet for juvenile turbot.

\section{Materials and Methods}

The experiments were conducted following the Animal Welfare Legislation ( $\$ 8$ Section 1 ) of the Ministry of Energy, Agriculture, the Environment and Rural Areas Schleswig-Holstein (Germany).

\section{Ingredients and experimental diets}

Nutrient composition, amino acid profiles and ANF's of dietary ingredients are shown in Table 1. The experimental diets were formulated by replacing FM protein in standard control feed to $50 \%$ (RPC 50) and to 75\% (RPC 75) with rapeseed protein concentrate (RPC). Remaining FM protein of RPC 50 and RPC 75 diets were further substituted by BMPC $\left(20-80 \mathrm{~g} \mathrm{~kg}^{-1}\right)$. Accordingly, diets were designated as RD, RPC 50/0, RPC 50/2, RPC 50/4, RPC 50/8, RPC 75/0, $\mathrm{RPC} 75 / 2$, RPC 75/4, RPC 75/8, (Table 2). BMPC was processed as byproduct of $\mathrm{CO}_{2}$ extraction of mussel oil by Flavex Naturextrakte $\mathrm{GmbH}$ (Rehlingen, Germany). Nutritional demands of turbot are fulfilled using diets formulated according to amino acid requirements $[38,39]$. The diets were pressed at a temperature of $60^{\circ} \mathrm{C}$ to pellets of $4 \mathrm{~mm}$ in diameter (L 14-175, Amandus Kahl, Reinbek, Germany) and were isonitrogenous $\left(55.6 \pm 0.85 \% \mathrm{CP} \mathrm{kg}^{-1} \mathrm{DM}\right)$ and isoenergetic $(22.51 \pm$ $\left.0.33 \mathrm{MJ} \mathrm{kg}^{-1} \mathrm{DM}\right)$ in their composition.

\section{Experimental setup}

The feeding trial was conducted at the experimental facilities of the Gesellschaft für Marine Aquakultur mbH (Büsum, Germany). A total of 496 juvenile turbot (obtained from MAXIMUS A/S, Gudnaesstrandvej 17, 7755 Bedsted Thy, Denmark) were stocked in 27 tanks $\left(175 \mathrm{~L}\right.$ each, bottom surface $\left.0.27 \mathrm{~m}^{2}\right)$ in a recirculation system ( $3.5 \mathrm{~m}^{3}$ water volume) equipped with mechanical and biological filters and a disinfection unit (UV-filter). Water exchange was approximately $540 \mathrm{~L} \mathrm{~kg}^{-1}$ feed and photoperiod was maintained at a 12L:12D-cycle. Water parameters were determined per day (salinity: $22.3 \pm 1.2$ $\mathrm{g} \mathrm{l}^{-1}$ HI 96822 Seawater Refractometer, Hanna Instruments Inc., Woonsocket-RI-USA; temperature: $18.7 \pm 0.4^{\circ} \mathrm{C}$; dissolved oxygen: 7.8 $\pm 0.3 \mathrm{mg} \mathrm{l}^{-1}$, Handy Polaris, Oxy Guard International A/S, Birkerod, Denmark; $\mathrm{NH}^{4+}: 0.2 \pm 0.1 \mathrm{mg} \mathrm{l}^{-1}, \mathrm{NO}^{2-}: 0.7 \pm 0.3 \mathrm{mg} \mathrm{l}^{-1}$, Microquant test kit, Merck KGaA, Darmstadt, Germany; pH-value: $7.7 \pm 0.2$, GMH 3530, Greisinger electronic GmbH, Regenstauf, Germany). For a $14 \mathrm{~d}$ acclimatization period, fish were fed with control diet once a day by hand until apparent satiation. After this period of acclimatization, fish were starved for 2 days and initial weight was determined individually (average initial body weight was $30.4 \pm 0.2 \mathrm{~g}$; stocking density: $1.68 \mathrm{~kg}$ $\mathrm{m}^{2}$ ). A total of 10 fish were sacrificed and stored at $-20^{\circ} \mathrm{C}$ for analysis of initial whole body composition. The remaining 486 fish were randomly restocked in triplicates to the tanks and fed twice a day by hand until apparent satiation over a period of 56 days. Unfed pellets were collected and re-counted for determination of daily feed intake.

\section{Sampling}

At the beginning and end of the experimental trial, biomass of each tank as well as individual weight and length were recorded. Condition factor (CF) and hepatosomatic index (HSI) were calculated. Daily feed intake (DFI, \% BW day ${ }^{-1}$ ) was recorded to evaluate the potential of 
Citation: Nagel F, Appel T, Rohde C, Kroeckel S, Schulz C (2017) Blue Mussel Protein Concentrate Versus Prime Fish Meal Protein as a Dietary Attractant for Turbot (Psetta maxima L.) Given Rapeseed Protein-based Diets. J Aquac Res Development S2: 012. doi:10.4172/2155-9546. S2-012

Page 3 of 7

\begin{tabular}{|c|c|c|c|c|c|c|c|c|c|}
\hline Variables & RD & RPC 50/0 & RPC 50/2 & RPC $50 / 4$ & RPC 50/8 & RPC 75/0 & RPC 75/2 & RPC 75/4 & RPC $75 / 8$ \\
\hline \multicolumn{10}{|c|}{ Ingredients ( $\mathrm{g} \mathrm{kg}^{-1}$ diet) } \\
\hline Herring meal $^{1}$ & 340 & 170 & 149 & 128 & 87 & 85 & 65 & 44 & 0 \\
\hline $\mathrm{RPC}^{2}$ & 0 & 171 & 171 & 171 & 171 & 256 & 256 & 256 & 256 \\
\hline Soyprotein concentrate ${ }^{3}$ & 150 & 150 & 150 & 150 & 150 & 150 & 150 & 150 & 150 \\
\hline Wheat starch ${ }^{4}$ & 128 & 108 & 107 & 106 & 103 & 99 & 97 & 96 & 95 \\
\hline Wheat gluten ${ }^{4}$ & 120 & 120 & 120 & 120 & 120 & 120 & 120 & 120 & 120 \\
\hline Corn gluten ${ }^{3}$ & 50 & 50 & 50 & 50 & 50 & 50 & 50 & 50 & 50 \\
\hline Blood meal $^{5}$ & 60 & 60 & 60 & 60 & 60 & 60 & 60 & 60 & 60 \\
\hline Mussel protein ${ }^{6}$ & 0 & 0 & 20 & 40 & 80 & 0 & 20 & 40 & 80 \\
\hline Shrimp meal ${ }^{1}$ & 40 & 40 & 40 & 40 & 40 & 40 & 40 & 40 & 40 \\
\hline Fish oil $^{1}$ & 62 & 81 & 83 & 85 & 89 & 90 & 92 & 94 & 99 \\
\hline Linseed oil $^{7}$ & 20 & 20 & 20 & 20 & 20 & 20 & 20 & 20 & 20 \\
\hline Vitamin/mineral mixture $^{8}$ & 10 & 10 & 10 & 10 & 10 & 10 & 10 & 10 & 10 \\
\hline $\mathrm{Ca}_{2} \mathrm{PO}_{4}{ }^{9}$ & 10 & 10 & 10 & 10 & 10 & 10 & 10 & 10 & 10 \\
\hline Titianium dioxide $^{10}$ & 10 & 10 & 10 & 10 & 10 & 10 & 10 & 10 & 10 \\
\hline \multicolumn{10}{|c|}{ Nutrient composition } \\
\hline Dry matter $\left(\mathrm{g} \mathrm{kg}^{-1}\right)$ & 931 & 936 & 931 & 938 & 933 & 944 & 942 & 939 & 941 \\
\hline Crude protein $\left(\mathrm{g} \mathrm{kg}^{-1} \mathrm{DM}\right)$ & 556 & 552 & 565 & 558 & 569 & 567 & 546 & 543 & 562 \\
\hline Crude lipid $\left(\mathrm{g} \mathrm{kg}^{-1} \mathrm{DM}\right)$ & 150 & 153 & 159 & 157 & 162 & 157 & 158 & 159 & 169 \\
\hline Crude ash $\left(\mathrm{g} \mathrm{kg}^{-1} \mathrm{DM}\right)$ & 116 & 100 & 98 & 97 & 94 & 92 & 90 & 89 & 86 \\
\hline Calcium ( $\left.\mathrm{g} \mathrm{kg}^{-1} \mathrm{DM}\right)$ & 24 & 19 & 19 & 18 & 15 & 16 & 15 & 14 & 12 \\
\hline Phosphorus ( $\left.\mathrm{g} \mathrm{kg}^{-1} \mathrm{DM}\right)$ & 15 & 14 & 14 & 13 & 12 & 13 & 13 & 12 & 11 \\
\hline $\mathrm{NfE}+$ crude fibre $\left(\mathrm{g} \mathrm{kg}^{-1} \mathrm{DM}\right)^{11}$ & 178 & 195 & 178 & 188 & 175 & 184 & 206 & 209 & 183 \\
\hline Gross energy (MJ kg $\left.{ }^{-1} \mathrm{DM}\right)$ & 22.3 & 22.6 & 22.7 & 22.7 & 22.8 & 22.7 & 21.6 & 22.4 & 22.8 \\
\hline \multicolumn{10}{|c|}{ Essential amino acids $\left(\mathrm{g} 100 \mathrm{~g}^{-1} \mathrm{CP}\right)$} \\
\hline Arginine & 4.0 & 4.2 & 4.2 & 4.2 & 4.2 & 4.3 & 4.3 & 4.3 & 4.3 \\
\hline Histidine & 1.9 & 2.0 & 2.0 & 2.0 & 2.0 & 2.1 & 2.1 & 2.1 & 2.1 \\
\hline Isoleucine & 2.7 & 2.8 & 2.8 & 2.8 & 2.7 & 2.8 & 2.8 & 2.8 & 2.8 \\
\hline Leucine & 5.7 & 5.9 & 5.8 & 5.8 & 5.8 & 5.9 & 5.9 & 5.9 & 5.9 \\
\hline Lysine & 3.9 & 3.8 & 3.8 & 3.7 & 3.7 & 3.7 & 3.7 & 3.7 & 3.6 \\
\hline Methionine + Cystine & 2.3 & 2.4 & 2.4 & 2.4 & 2.4 & 2.4 & 2.5 & 2.5 & 2.5 \\
\hline Phenylalanine & 3.3 & 3.4 & 3.4 & 3.4 & 3.4 & 3.5 & 3.5 & 3.5 & 3.5 \\
\hline Threonine & 2.6 & 2.6 & 2.6 & 2.6 & 2.6 & 2.6 & 2.6 & 2.7 & 2.7 \\
\hline Valine & 3.5 & 3.6 & 3.5 & 3.5 & 3.5 & 3.6 & 3.6 & 3.6 & 3.5 \\
\hline \multicolumn{10}{|c|}{ Anti-nutritional factors } \\
\hline Glucosinolates $\left(\mu \mathrm{mol} \mathrm{g}^{-1}\right)$ & 0.03 & 0.07 & 0.07 & 0.07 & 0.07 & 0.08 & 0.08 & 0.08 & 0.08 \\
\hline Phytic acid $\left(\mathrm{g} \mathrm{kg}^{-1}\right)$ & 3.54 & 7.4 & 7.4 & 7.4 & 7.4 & 9.3 & 9.3 & 9.3 & 9.3 \\
\hline $\begin{array}{l}\text { Sinapinic acid and sinapinic acid ester } \\
\qquad\left(\mathrm{g} \mathrm{kg}^{-1}\right)\end{array}$ & 0.00 & 4.1 & 4.1 & 4.1 & 4.1 & 6.15 & 6.15 & 6.15 & 6.15 \\
\hline
\end{tabular}

IVFC GmbH, Cuxhaven, Germany, ${ }^{2} \mathrm{HELM}$ AG, Business Unit Animal Nutrition, Hamburg, Germany, ${ }^{3}$ Cargill Deutschland GmbH, Krefeld, Germany, ${ }^{4}$ Kröner Stärke $\mathrm{GmbH}$, Ibbenbüren, Germany, ${ }^{5}$ Euroduna-Technologies $\mathrm{GmbH}$, Barmstedt, Germany, ${ }^{6}$ Frozen blue mussel meat from Royal Frysk Muscheln GmbH, Emmelsbüll-Horsbüll, Germany; freeze-dried meal manufactured by the Costal Research \& Management, Kiel, Germany, Processing of blue mussel protein concentrate (by-product of $\mathrm{CO}_{2}$-oil extraction) by Flavex Naturextrakte $\mathrm{GmbH}$, Rehlingen, Germany, ${ }^{7}$ Makana Produktion und Vertrieb GmbH, Offenbach a.d. Queich, Germany, ${ }^{8}$ Vitamin and mineral mixture, Vitfoss, Gråsten, Denmark, ${ }^{9}$ Lehmann \& Voss \& Co, Hamburg, Germany, ${ }^{10} \mathrm{Kronos}$ Titan $\mathrm{GmbH}$, Leverkusen, Germany, ${ }^{11}$ Nitrogen-free extracts $\left(\mathrm{NfE}\right.$, g kg $\left.{ }^{-1} \mathrm{DM}\right)=1000$ (crude protein + crude lipid + crude ash + crude fiber)

Table 2: Ingredients, nutrient composition and dietary amino acid profile of experimental diets (RD, Reference diet; RPC 50 or 75 indicate $50 \%$ or $75 \%$ fish meal protein replacement with rapeseed protein concentrate; 0-8 indicate inclusion of blue mussel protein concentrate with 0-80 $\mathrm{g} \mathrm{kg}^{-1} \mathrm{diet}$ ).

BMPC as feed attractant. Furthermore, for each treatment, mortalities, specific growth rate (SGR, \% BW day ${ }^{-1}$ ) and feed conversion ratio (FCR) were calculated as average of the triplicates according to formulae given in Table 3. A representative sample of three fish per each tank was taken for analysis of final whole body composition. Protein efficiency ratio (PER) and protein productive value (PPV) were calculated according to formulae given in Table 3 .

\section{Dietary and whole fish body nutrient composition}

Diets and homogenised fish body samples were analyzed for dry matter (DM), crude ash, crude protein (CP), crude lipid (CL) and gross energy according to EU guideline (EC/152/2009) [40]. DM was determined after drying at $105^{\circ} \mathrm{C}$ until weight remained constant and ash content after $4 \mathrm{~h}$ incineration at $550^{\circ} \mathrm{C}$ with a combustion oven $(\mathrm{P} 300$; Nabertherm, Lilienthal, Germany). CP content ( $\mathrm{N}$ x 6.25) was analyzed by the Kjeldahl method (InKjel 1225 M, WD 30; Behr, Düsseldorf, Germany), CL content after hydrolysis with hydrochloric acid (for experimental diets but not for whole body composition) followed by a petroleum ether extraction with a Soxleth extraction system (R $106 \mathrm{~S}$; Behr). Gross energy was measured in a bomb calorimeter (C 200; IKA, Staufen, Germany). Sum of nitrogen free extracts (NfE) and crude fibre were calculated on DM basis by $100-(\% \mathrm{CP}+\% \mathrm{CL}+\%$ ash). ANFs have been analyzed as follows: Glucosinolates and phytic acid were determined by the ÖHMI Analytic GmbH (Magdeburg, Germany) in accordance to the methods registered in the Gazette of the EC (1864/90 Nr. L 170/28 and SAA A 006). Mustard seed oil was determined by 
Citation: Nagel F, Appel T, Rohde C, Kroeckel S, Schulz C (2017) Blue Mussel Protein Concentrate Versus Prime Fish Meal Protein as a Dietary Attractant for Turbot (Psetta maxima L.) Given Rapeseed Protein-based Diets. J Aquac Res Development S2: 012. doi:10.4172/2155-9546. S2-012

Page 4 of 7

\begin{tabular}{|c|c|c|c|c|c|c|c|c|c|}
\hline \multirow{2}{*}{ Variables } & & & \multicolumn{7}{|c|}{ Experimental diet } \\
\hline & RD & RPC 50/0 & RPC 50/2 & RPC 50/4 & RPC 50/8 & RPC $75 / 0$ & RPC $75 / 2$ & RPC $75 / 4$ & RPC $75 / 8$ \\
\hline IBW $^{1}$ & $30.2 \pm 0.1$ & $30.3 \pm 0.6$ & $30.6 \pm 0.5$ & $30.6 \pm 0.3$ & $30.4 \pm 0.6$ & $30.0 \pm 0.4$ & $30.7 \pm 0.3$ & $30.6 \pm 0.5$ & $30.5 \pm 0.4$ \\
\hline FBW $^{2}$ & $95.6 \pm 11.1^{\mathrm{A}}$ & $84.6 \pm 11.0^{\mathrm{A}}$ & $87.2 \pm 12.9$ & $91.9 \pm 12.1$ & $94.4 \pm 11.8$ & $66.0 \pm 11.4^{\mathrm{B}}$ & $73.1 \pm 9.0$ & $71.7 \pm 10.2$ & $73.9 \pm 11.4$ \\
\hline DFI $\left[\% B W \text { day }^{-1}\right]^{3}$ & $1.65 \pm 0.02^{A}$ & $1.51 \pm 0.11^{\mathrm{A}}$ & $1.49 \pm 0.07$ & $1.51 \pm 0.09$ & $1.53 \pm 0.07$ & $1.25 \pm 0.04^{\mathrm{B}}$ & $1.32 \pm 0.02$ & $1.31 \pm 0.05$ & $1.32 \pm 0.02$ \\
\hline SGR $\left[\% \text { BW } \text { day }^{-1}\right]^{4}$ & $2.15 \pm 0.07^{A}$ & $1.92 \pm 0.09^{A}$ & $1.95 \pm 0.09$ & $2.04 \pm 0.14$ & $2.11 \pm 0.14$ & $1.49 \pm 0.09^{B}$ & $1.63 \pm 0.06$ & $1.61 \pm 0.06$ & $1.68 \pm 0.09$ \\
\hline FCR $^{5}$ & $0.77 \pm 0.02^{A}$ & $0.78 \pm 0.05^{A}$ & $0.77 \pm 0.01$ & $0.74 \pm 0.02$ & $0.73 \pm 0.01$ & $0.84 \pm 0.02^{\mathrm{B}}$ & $0.81 \pm 0.02$ & $0.82 \pm 0.01$ & $0.79 \pm 0.03$ \\
\hline
\end{tabular}

IInitial body weight (g); ${ }^{2}$ Final body weight (g); ${ }^{3}$ Daily feed intake (\% BW day $\left.{ }^{-1}\right) ;{ }^{4}$ Specific growth rate $\left(\%\right.$ BW day $\left.{ }^{-1}\right)=\left[\ln (\mathrm{FBW})-\ln (\right.$ IBW) $] /$ feeding days $\times 100 ;{ }^{5} \mathrm{Feed}$ conversion ratio $=$ Feed intake $(\mathrm{g} \mathrm{DM}) /$ weight gain $(\mathrm{g})$

Table 3: Growth performance of juvenile turbot fed experimental diets for 56 days. Values (mean \pm S.D.) with different superscript letters indicate significant differences of fish meal protein replacement by rapeseed protein concentrate (comparison of reference diet (RD) with RPC 50/0 and RPC 75/0 diets). No significance differences were detected within the RPC 50 or RPC 75 group when BMPC was included (one-way ANOVA Tukey's HSD post hoc test; P>0.05).
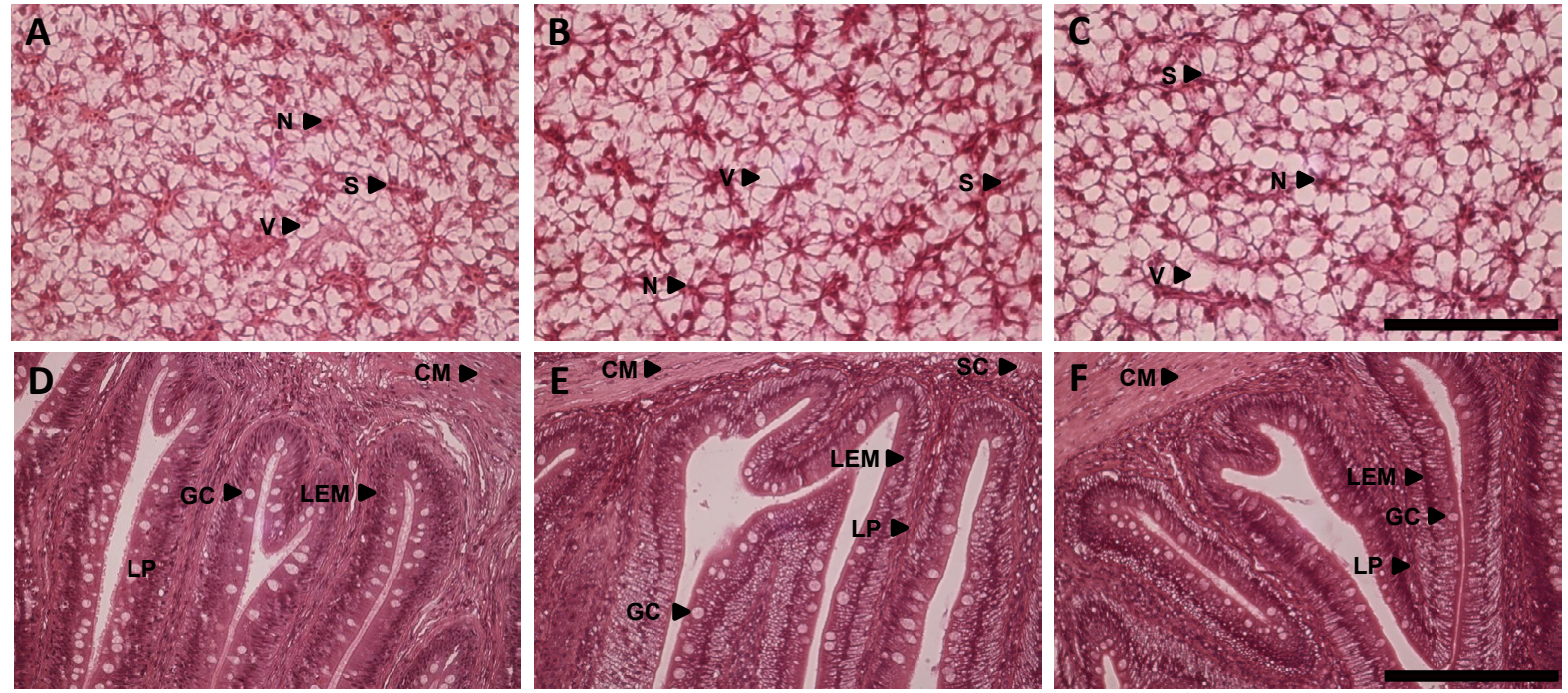

Figure 1: Histological sections (Hematoxylin and eosin staining) of the liver (A-C) and midgut (D-F) of juvenile turbot fed experimental diets. Images are representative for fish fed reference diet (A,D), RPC 50 and RPC 75 diets with 0-20 $\mathrm{g} \mathrm{kg}^{-1}$ blue mussel protein concentrate (B,E) and RPC 50 and RPC 75 diets with $40-80 \mathrm{~g} \mathrm{~kg}{ }^{-1}$ blue mussel protein concentrate (C,F). Black arrowheads point to S: Sinusoid; V: Vacuole; N: Nucleus; GC: Goblet cell; LEM: Lamina epithelialis mucosae; LP: Lamina propria; SC: Stratum compactum; CM: Tunica muscularis. Scale bar $=100 \mu \mathrm{m}(A-C), 200 \mu \mathrm{m}(D-F)$.

the LUFA-ITL GmbH (Kiel, Germany; analogue VDLUFA III 16.6.1) and tannin concentration was detected by Phytolab GmbH \& Co Kg. (Vestenbergsgreuth, Germany; Ph. Eur. 5.0 2.8.14 v). Sinapinic acid and sinapinic acid ester were determined by HPLC analysis at the A.C.T. FOODs GmbH (Bad Fallingbostel, Germany). Detailed ANF analysis of RPC is shown in Table 1. Dietary concentration of the most prominent ANF phytic acid was then calculated to be $2.0 \mathrm{~g} \mathrm{~kg}^{-1}$ feed (control group) - $13.0 \mathrm{~g} \mathrm{~kg}^{-1}$ feed (RPC 75/0; Table 2).

\section{Histology}

Sections of liver and mid gut were randomly sampled from three fish of each tank. After fixation in $4 \%$ paraformaldehyde, the samples were dehydrated in a graded ethanol series and embedded in paraffin. Slices were cut at $3 \mu \mathrm{m}$ and stained with Haematoxylin-eosin. Labelling was visualized by light microscopy using an Olympus CKX 41 microscope (Olympus Deutschland GmbH, Hamburg, Germany) equipped with a Canon EOS 500D digital camera.

\section{Statistical analysis}

Statistical analyses were performed using SPSS 17.0 for Windows (SPSS Inc., Chicago, USA). Data were checked for normal distribution by the Kolmogorov Smirnov test. Effects of fish meal replacement by RPC (comparison of reference diet, RPC 50/0 and RPC 75/0) or impact of BMPC application (within the RPC 50 or RPC 75 treatments) on growth performance, nutrient utilization and health parameters were analysed by one-way analysis of variance (ANOVA) followed by Tukey's HSD post hoc-test for homogeneous variances (Levene's test). Significance was attributed to $\mathrm{P}<0.05$.

\section{Results}

During the experiment no mortality of fish was recorded. 50\% of dietary FM replacement (by RPC, inclusion $171 \mathrm{~g} \mathrm{~kg}^{-1}$ ) did not caused significant reduction in performance of juvenile turbot $(\mathrm{P}>0.05)$. However, DFI, SGR and FCR were significantly affected by FM substitution of $75 \%$ (RPC inclusion $256 \mathrm{~g} \mathrm{~kg}^{-1} ; \mathrm{P}<0.05$, Table 3). Incorporation of blue mussle protein concentrate (BMPC) into both, RPC 50 and RPC 75 diets did not enhance DFI or SGR of fish $(P>0.05$, one-way ANOVA, Table 3). With BMPC incorporation (20-80 g kg $\left.{ }^{1}\right)$, FCR showed a slight improvement without reaching statistical significance $(\mathrm{P}>0.05)$.

Hepatosomatic Index (HSI) was unaffected by RPC and BMPC incorporation $(\mathrm{P}>0.05)$. However, a tendency towards increased liver size was observed for fish fed $40-80 \mathrm{~g} \mathrm{BMPC} \mathrm{kg}^{-1}$ diets. Histopathological investigation of this liver tissue showed slighty hypervacuolated hepatocytes (Figures 1A-1C). For the other treatments a normal vacuolization was observed (Figures $1 \mathrm{~A}$ and $1 \mathrm{~B}$ ). Among the groups neither inflammatory nor degenerative changes were determined for the intestine (Figures 1D-1F). 
Citation: Nagel F, Appel T, Rohde C, Kroeckel S, Schulz C (2017) Blue Mussel Protein Concentrate Versus Prime Fish Meal Protein as a Dietary Attractant for Turbot (Psetta maxima L.) Given Rapeseed Protein-based Diets. J Aquac Res Development S2: 012. doi:10.4172/2155-9546. S2-012

Page 5 of 7

\begin{tabular}{|c|c|c|c|c|c|c|c|c|c|c|}
\hline & RD & RPC 50/0 & RPC 50/2 & RPC 50/4 & RPC 50/8 & RPC 75/0 & RPC 75/2 & RPC 75/4 & RPC 75/8 \\
\hline \multicolumn{7}{|c|}{ Proximate body composition (\% wet weight) } \\
\hline Dry matter & $24.7 \pm 0.9$ & $25.1 \pm 0.4$ & $24.7 \pm 0.7$ & $25.2 \pm 0.6$ & $24.4 \pm 2.7$ & $23.9 \pm 0.5$ & $23.5 \pm 0.9$ & $24.2 \pm 0.4$ & $24.5 \pm 0.5$ \\
\hline Crude protein & $15.8 \pm 0.4$ & $16.0 \pm 0.1$ & $15.8 \pm 0.2$ & $15.9 \pm 0.2$ & $15.3 \pm 1.5$ & $15.9 \pm 0.1$ & $15.6 \pm 0.7$ & $15.7 \pm 0.1$ & $15.7 \pm 0.1$ \\
\hline Crude lipid & $5.5 \pm 0.4$ & $5.9 \pm 0.3$ & $5.7 \pm 0.5$ & $6.1 \pm 0.6$ & $6.3 \pm 1.1$ & $4.9 \pm 0.6$ & $4.9 \pm 0.3$ & $5.5 \pm 0.3$ & $5.9 \pm 0.5$ \\
\hline Ash & $3.4 \pm 0.1$ & $3.3 \pm 0.1^{\mathrm{a}}$ & $3.2 \pm 0.1^{\mathrm{ab}}$ & $3.2 \pm 0.0^{\mathrm{ab}}$ & $2.8 \pm 0.2^{\mathrm{b}}$ & $3.1 \pm 0.1^{\mathrm{a}}$ & $3.0 \pm 0.1^{\mathrm{ab}}$ & $3.0 \pm 0.1^{\mathrm{ab}}$ & $2.8 \pm 0.0^{\mathrm{b}}$ \\
\hline \multicolumn{7}{|c|}{ Biometric parameters } \\
\hline Gross energy (MJ kg-1 DM) & $23.3 \pm 0.4$ & $23.4 \pm 0.2$ & $23.4 \pm 1.0$ & $23.4 \pm 0.3$ & $24.1 \pm 0.1$ & $22.9 \pm 0.2$ & $23.2 \pm 1.0$ & $23.2 \pm 0.3$ & $23.8 \pm 0.2$ \\
\hline CF $^{1}$ & $2.23 \pm 0.06$ & $2.05 \pm 0.11$ & $2.17 \pm 0.11$ & $2.21 \pm 0.11$ & $2.25 \pm 0.13$ & $2.10 \pm 0.06$ & $2.14 \pm 0.04$ & $2.17 \pm 0.07$ & $2.27 \pm 0.09$ \\
\hline HSI $^{2}$ & $1.97 \pm 0.24$ & $1.94 \pm 0.28$ & $2.18 \pm 0.18$ & $2.41 \pm 0.49$ & $2.44 \pm 0.27$ & $2.08 \pm 0.27$ & $2.18 \pm 0.19$ & $2.41 \pm 0.17$ & $2.45 \pm 0.44$ \\
\hline
\end{tabular}

Table 4: Proximate whole body composition and biometric parameters of turbot fed experimental diets for 56 days. Values (mean \pm S.D.) with different superscript letters indicate significant differences within the RPC 50 or the RPC 75 group with $0 \%, 2 \%, 4 \%$ or $8 \%$ BMPC inclusion (one way ANOVA, Tukey's HSD-post hoc test, P<0.05).

\begin{tabular}{|c|c|c|c|c|c|c|c|c|c|c|c|}
\hline \multirow{2}{*}{ Variables } & \multicolumn{4}{|c|}{} & \multicolumn{4}{|c|}{ Experimental diet } \\
\cline { 2 - 9 } & RD & RPC 50/0 & RPC 50/2 & RPC 50/4 & RPC 50/8 & RPC 75/0 & RPC 75/2 & RPC 75/4 & RPC 75/8 \\
\hline PER ${ }^{1}$ & $2.34 \pm 0.05$ & $2.31 \pm 0.15$ & $2.31 \pm 0.02$ & $2.42 \pm 0.07$ & $2.42 \pm 0.05$ & $2.24 \pm 0.06$ & $2.26 \pm 0.05$ & $2.26 \pm 0.03$ & $2.25 \pm 0.08$ \\
\hline PPV $^{2}$ & $38.6 \pm 0.7$ & $39.1 \pm 2.5$ & $38.43 \pm 1.1$ & $40.4 \pm 1.2$ & $38.1 \pm 5.5$ & $36.0 \pm 1.0$ & $37.4 \pm 3.3$ & $37.8 \pm 0.2$ & $37.6 \pm 1.0$ \\
\hline
\end{tabular}

${ }^{1}$ Protein efficiency ratio $=$ Body weight gain $(\mathrm{g}) /$ protein intake $(\mathrm{g}) ;{ }^{2}$ Protein productive value $=100 \times[($ crude protein final fish $(\%) \times$ biomass final tank weight $(\mathrm{g}))-(\mathrm{crude}$ protein initial fish $(\%) \times$ biomass initial tank weight $(\mathrm{g}))] /($ crude protein diet $(\mathrm{g})$ )

Table 5: Protein efficiency ratio (PER) and protein productive value (PPV) of turbot. No significant differences were detected among the groups (one way ANOVA, Tukey's post hoc test, $\mathrm{P}>0.05)$

Proximate whole body composition of fish at the beginning and at the end of the feeding trial is presented in Table 4. Treatments showed no significant changes for most of the analyzed parameters compared to control with exception of crude ash content, which declined significantly within the RPC 50 and RPC 75 groups $(\mathrm{P}<0.05)$. Crude lipid content tended to increase with incorporation of $\mathrm{BMPC}(\mathrm{P}>0.05)$. The protein efficiency ratio (PER) and protein productive value (PPV) remained unaffected among all treatments $(\mathrm{P}>0.05)$, suggesting no impact of diet composition on protein retention (Table 5 ).

\section{Discussion}

Previous findings with turbot showed that DFI and SGR decreased significantly when FM protein was substituted by RPC to levels below $300 \mathrm{~g} \mathrm{FM} \mathrm{kg}^{-1}$ diet $[10,19,37]$. In the present study, this concentration was further reduced below $170 \mathrm{~g} \mathrm{FM} \mathrm{kg}^{-1}$ feed (RPC 50 diets) expecting a reduced feed intake of turbot to investigate the potential of BMPC as feed stimulant. However, RPC 50 diets did not affect neither DFI nor FCR significantly; fish maintained on a good performance level compared with turbot of same size and origin at previous trials [10,37], which emphasises the nutritional quality of test diets, but restricted the validity of the RPC 50/0 diets to evaluate the full potential of BMPC as feed stimulant. However, FM substitution towards $\leq 85 \mathrm{~g} \mathrm{FM} \mathrm{kg}^{-1}$ feed (RPC 75 diets) triggered a significant decline in the general performance including DFI of juvenile turbot. Hence, it was suitable to utilize these diets to evaluate the BMPC-stimulating effect on turbot.

Under natural conditions, juvenile turbot of comparable size as in this study forage mainly on molluscs and annelids [41], which contain higher amounts of feeding stimulants [42,43]. The utilization of blue mussels either fresh, as freeze-dried meal or extracts in diets of several species provided conflicting results [27,28,32,34-37]. For example, the application of mussel meal failed to increase DFI in experimental trials with Japanese flounder [28,32,33], juvenile barramundi (Lates calcarifer) [44] or rainbow trout [8]. On the contrary, Kikuchi [35] and Nagel et al. [37] have observed increased feed intake of flounder and turbot when fed with mussel meal-supplemented diets, suggesting its potential use to alleviate plant protein-derived palatability impairments. In proteinextracts of the blue mussel glycine and alanine together with betaine and/or taurine have been identified to improve feeding behavior of common sole [29] and Japanese flounder [36]. Chemical analogue confirmed the potential of mussel amino acids acting as feed attractants $[29,43]$. In the present investigation, BMPC (with a comparable amino acid profile to FM protein) was utilized to further replace FM protein totally in the RPC 75/8 treatment. In contrast to increased DFI of turbot fed diets where wheat gluten [37] or soybean meal [35] were replaced by mussel meal, no DFI improvements were detected when turbot fed diets of BMPC-mediated FM protein reduction in this study. The latter has been confirmed by Kikuchi and Sakguchi [32], who utilized mussel meal as total FM replacer. This indicates that BMPC is comparable with high quality FM protein in term of palatability and feed acceptance. Furthermore, SGR showed comparable values and feed conversion tend to be improved for fish fed with BMPC supplemented diets. This FCR-trend is in accordance with findings by Anagnostidis et al. [45], who suggested a better nutrient utilization of mussel protein compared to FM protein. Although our PPVs (36.0\% to 40.4\%) are in line [6] or slightly above recently reported protein retention levels for juvenile turbot [46], indicating an efficient protein utilization of turbot [47], these values do not support the BMPC-driven trend in the FCR values for RPC 75 diets. Experimental diets were balanced in terms of crude protein and lipid content, which could not exclude varying values on the digestibility level. This issue could influence feed intake, protein digestion and absorption as well as intestinal evacuation rates [48].

In turbot, feed intake and growth performance were in accordance with unaffected CF and HSI. In contrast, investigations [21,49] showed decreased CF (but unaffected HSI) when FM protein was progressively substituted with soybean protein concentrate. Considering the HSI, Regost et al. [6] and Fournier et al. [50] had determined a significant decline in HSI when turbot have been fed with diets of high plant protein incorporation level. This might have been affected by reduced protein and energy intake and less efficient conversion and retention needed for growth [46]. Consequently, the lack of ingested energy could have been balanced by mobilization of liver-stored energy.

Histopathological investigations of gut and liver are rarely correlated with the dietary utilization of animal proteins. Recently, it has been reported that either a 30\% incorporation of blood and feather meal or a $60 \%$ FM protein substitution with an animal protein blend (a mixture of poultry, meat, bone, blood and hydrolyzed feather meal) 
Citation: Nagel F, Appel T, Rohde C, Kroeckel S, Schulz C (2017) Blue Mussel Protein Concentrate Versus Prime Fish Meal Protein as a Dietary Attractant for Turbot (Psetta maxima L.) Given Rapeseed Protein-based Diets. J Aquac Res Development S2: 012. doi:10.4172/2155-9546. S2-012

Page 6 of 7

induced hepatic steatosis in Japanese seabass Lateolabrax japonicus $[51,52]$, which was indicated by an increased HSI and fat accumulation in the liver. Whether fat accumulation or increased density of intracytoplasmatic lipidic droplets [53] is caused by a deficiency of lipitrophic factors or elevated lipogenic enzymes was beyond the scope of the present study. However, liver of fish fed diets with 40-80 $\mathrm{g}$ BMPC $\mathrm{kg}^{-1}$ diet showed slight hypervacuolated hepatocytes. This might be an early indication for incipient hepatic steatosis [51,52].

\section{Conclusion}

BMPC failed to stimulate the feed intake of turbot when FM protein was substituted in already RPC-based diets. However, the maintenance of performance within the test diet treatments revealed a nutritional value comparable to prime FM protein. It indicates BMPC as ingredient with the potential to further reduce the FM protein content in aquafeeds of carnivorous fish.

\section{Acknowledgements}

We gratefully acknowledge the assistance of Kevin Stiller, Biniam S. Fitwi, Anja Winkelbach, Sven Wuertz and Simon F. Klatt during experimental trial. We also thank Chris van Bussel for critical discussion and comments on the manuscript. We further thank Stefan Meyer for assistance with statistical analysis. The authors wish to thank Andreas Lemme (Evonik Industries AG, Hanau, Germany) for analysing amino acid profile of ingredients and experimental diets. The project was supported by funds of the Federal Ministry of Food, Agriculture and Consumer Protection (BMELV) based on a decision of the Parliament of the Federal Republic of Germany via the Federal Office for Agriculture and Food (BLE) under the innovation support programme. We acknowledge financial support by Land Schleswig-Holstein within the funding programme Open Access Publikationsfonds.

\section{References}

1. Hua K, Bureau DP (2012) Exploring the possibility of quantifying the effects of plant protein ingredients in fish feeds using meta-analysis and nutritional model simulation-based approaches. Aquaculture 356: 284-301.

2. Webster CD, Tidwell JH, Goodgame LS, Yancey DH, Mackey L (1992) Use of soybean meal and distillers grains with solubles as partial or total replacement of fish meal in diets for channel catfish, Ictalurus punctatus. Aquaculture 106: 301-309.

3. Kaushik SJ, Cravedi JP, Lalles JP, Sumpter J, Fauconneau B, et al. (1995) Partial or total replacement of FM by soybean protein on growth, protein utilization, potential estrogenic or antigenic effects, cholesterolemia and flesh quality in rainbow trout (Oncorhynchus mykiss). Aquaculture 133: 257-274.

4. Slawski H, Adem H, Tressel RP, Wysujack K, Koops U, et al. (2012) Total fish meal replacement with rapeseed protein concentrate in diets fed to rainbow trout (Oncorhynchus mykiss, Walbaum). Aquacult Int 20: 443-453.

5. Slawski H, Nagel F, Wysujack K, Balke DT, Franz P, et al. (2013) Total fish meal replacement with rapeseed protein isolate in diets fed to rainbow trout (Oncorhynchus mykiss, Walbaum). Aquacult Nutr 19: 535-542.

6. Regost C, Arzel J, Kaushik SJ (1999) Partial or total replacement of fish meal by corn gluten meal in diet for turbot (Psetta maxima). Aquaculture 180: 99-117.

7. Hansen AC, Rosenlund G, Karlsen $\varnothing$, Koppe W, Hemre GI (2007) Tota replacement of fish meal with plant proteins in diets for Atlantic cod (Gadus morhua L.) - Effects on growth and protein retention. Aquaculture 272: 599-611.

8. Tusche K, Berends K, Wuertz S, Susenbeth A, Schulz C (2011) Evaluation of feed attractants in potato protein concentrate based diets for rainbow trout (Oncorhynchus mykiss). Aquaculture 321: 54-60.

9. Nagel F, Slawski H, Adem H, Tressel RP, Wysujack K, et al. (2012a) Albumin and globulin rapeseed protein fractions as fish meal alternative in diets fed to rainbow trout (Oncorhynchus mykiss W.). Aquaculture 354-355: 121-127.

10. Nagel F, von Danwitz A, Tusche K, Kroeckel S, Van Bussel CGJ, et al (2012b) Nutritional evaluation of rapeseed protein isolate as fish mea substitute for juvenile turbot (Psetta maxima L.) - Impact on growth performance, body composition, nutrient digestibility and blood physiology. Aquaculture 356: 357-364.

11. Francis G, Makkar HPS, Becker K (2001) Antinutritional factors present in plant-derived alternate fish feed ingredients and their effects in fish. Aquaculture 199: 197-227.
12. Collins SA, Desai AR, Mansfield GS, Hill JE, Van Kessel AG, et al. (2012) The effect of increasing inclusion rates of soybean, pea and canola meals and their protein concentrates on the growth of rainbow trout: Concepts in diet formulation and experimental design for ingredient evaluation. Aquaculture 344-349: 90-99.

13. Mwachireya SA, Beames RM, Higgs DA, Dosanjh BS (1999) Digestibility of canola protein products derived from the physical, enzymatic and chemical processing of commercial canola meal in rainbow trout, Oncorhynchus mykiss (Walbaum) held in fresh water. Aquacult Nutr 5: 73-82.

14. Thiessen DL, Maenz DD, Newkirk RW, Classen HL, Drew MD (2004) Replacement of fish meal by canola protein concentrate in diets fed to rainbow trout (Oncorhynchus mykiss). Aquacult Nutr 10: 379-388.

15. Slawski H, Adem H, Tressel RP, Wysujack K, Koops U, et al. (2011a) Replacement of fish meal with rapeseed protein concentrate in diets fed to common carp (Cyprinus carpio L.). ISR J Aquacult-Bamidgeh 63: 605-611.

16. Slawski H, Adem H, Tressel RP, Wysujack K, Koops U, et al. (2011b) Replacement of fish meal with rapeseed protein concentrate in diets fed to wels catfish (Silurus glanis L.). Aquacult Nutr 17: 605-612.

17. Burel C, Boujard T, Tulli F, Kaushik SJ (2000a) Digestibility of extruded peas extruded lupin, and rapeseed meal in rainbow trout (Oncorhynchus mykiss) and turbot (Psetta maxima). Aquaculture 188: 285-298.

18. Burel C, Boujard T, Kaushik SJ, Boeuf G, Van Der Geyten S, et al. (2000b) Potential of plant-protein sources as fish meal substitutes in diets for turbot (Psetta maxima): growth, nutrient utilisation and thyroid status. Aquaculture 188: 363-382.

19. Slawski H, Adem H, Tressel RP, Wysujack K, Kotzamanis $Y$, et al. (2011c) Austausch von Fischmehl durch Rapsproteinkonzentrat in Futtermitteln fü Steinbutt (Psetta maxima L.). Züchtungskunde 83: 51-60.

20. Day OJ, Howell BR, Jones DA (1997) The effect of dietary hydrolysed fish protein concentrate on the survival and growth of juvenile Dover sole, Solea solea (L.), during and after weaning. Aquacult Res 28: 911-921.

21. Day OJ, Plascencia GHG (2000) Soybean protein concentrate as a protein source for turbot Scophthalmus maximus L. Aquacult Nutr 6: 221-228.

22. Papatryphon E, Soares Jr JH (2000) The effect of dietary feeding stimulants on growth performance of striped bass, Morone saxatilis, fed-a-plant feedstuffbased diet. Aquaculture 185: 329-338.

23. Gaylord TG, Teague AM, Barrows FT (2006) Taurine supplementation of allplant protein diets for rainbow trout (Oncorhynchus mykiss). J World Aquacult Soc 37: 509-517.

24. Tiril SU, Alagil F, Yagci FB, Aral O (2008) Effects of betaine supplementation on plant protein based diets on feed intake and growth performance in rainbow trout (Oncorhynchus mykiss). ISR J Aquacult-Bamidgeh 60: 57-64.

25. Kader MdA, Bulbul M, Shunsuke K, Ishikawa M, Yokoyama S, et al. (2012) Effect of complete replacement of fishmeal by dehulled soybean meal with crude attractants supplementation in diets for red sea bream, Pagrus major. Aquaculture 350-353: 109-116

26. FAO (2012) Fisheries and Aquaculture Department, Food and Agriculture Organization of the United Nations. The State of World Fisheries and Aquaculture. Rome, Italy.

27. Berge GM, Austreng E (1989) Blue mussel in feed for rainbow trout. Aquaculture 81: 79-90.

28. Kikuchi K, Furuta T (2009a) Inclusion of blue mussel extract in diets based on fish and soybean meals for tiger puffer Takifugu rubripes. Fish Sci 75: 183-189.

29. Mackie AM, Adron JW, Grant PT (1980) Chemical nature of feeding stimulants for juvenile Dover sole, Solea solea (L.). J Fish Biol 16: 701-708.

30. Grave H, Schultz A, Van Thielen R (1979) The influence of blue mussel, Mytilus edulis, and krill, Euphausia superba, on growth and proximate composition of rainbow trout, Salmo gairdneri. Proceedings of world symposium on finfish nutrition and fish feed technology, Heenemann, Berlin.

31. Kitamura H, Mizutani H, Dotsu $Y$ (1981) Rearing experiment on young of some fishes and decapodid crustaceans fed on the meat of the common mussel, Mytilus edulis gallo provincialis. Marine Fouling 3: 23-27.

32. Kikuchi K, Sakaguchi I (1997) Blue mussel as an ingredient in the diet of juvenile Japanese flounder. Fish Sci 63: 837-838. 
Citation: Nagel F, Appel T, Rohde C, Kroeckel S, Schulz C (2017) Blue Mussel Protein Concentrate Versus Prime Fish Meal Protein as a Dietary Attractant for Turbot (Psetta maxima L.) Given Rapeseed Protein-based Diets. J Aquac Res Development S2: 012. doi:10.4172/2155-9546. S2-012

33. Kikuchi K, Furuta T (2009b) Use of defatted soybean meal and blue mussel meat as substitute for fish meal in the diet of tiger puffer, Takifugu rubripes. $J$ World Aquacult Soc 40: 472-482.

34. Fonds M, Drinkwaard B, Resink JW, Eysink GGJ, Toet W (1989) Measurements of metabolism, food intake and growth of Solea solea (L.) fed with mussel meat or with dry food. Aquaculture: a biotechnology in progress. 2: 851-874

35. Kikuchi K (1999) Use of defatted soybean meal as a substitute for fish meal in diets of Japanese flounder (Paralichthys olivaceus). Aquaculture 179: 3-11.

36. Kikuchi K, Ueda A, Sugita H, Takeda S (2002) Effect of dietary inclusion of blue mussel extract on growth and body composition of Japanese flounder Paralichthys olivaceus. J World Aquacult Soc 33: 41-47.

37. Nagel F, Von Danwitz A, Schlachter M, Kroeckel S, Wagner C, et al. (2014) Blue mussel meal as feed attractant in rapeseed protein-based diets for turbot (Psetta maxima L.). Aquacult Res 45: 1964-1978.

38. Kaushik SJ (1998) Whole body amino acid composition of European seabass (Dicentrarchus labrax), gilthead seabream (Sparus aurata) and turbot (Psetta maxima) with an estimation of their IAA requirement profiles. Aquat Liv Res 11: $355-358$

39. Peres H, Olivia-Teles A (2008) Lysin requirement and efficiency of lysine utilization in turbot (Scophthalmus maximus) juveniles. Aquaculture 275: 283290.

40. European Union (2009) Commission regulation (EC) No 152/2009 of 27 January 2009 Laying down the methods of sampling and analysis for the official control of feed. Official Journal of the European Union 52: 1-130.

41. De Groot SJ (1971) On the interrelationships between morphology of the alimentary tract, food and feeding behavior in flatfishes (Pisces: Pleuronectiformes). Neth J Sea Res 5: 121-196.

42. Mackie AM, Adron JW (1978) Identification of inosine and inosine 5'-monophosphate as the gustatory feeding stimulants for turbot Scophthalmus maximus. Comp Biochem Physiol 60: 79-83.

43. Tandler A, Berg BA, Kissil G, Mackie AM (1982) Effect of food attractants on appetite and growth rate of gilthead bream, Sparus aurata L. J Fish Biol 20: 673-681.
44. Nankervis L, Southgate PC (2006) An integrated assessment of gross marine protein sources used in formulated microbound diets for barramundi (Lates calcarifer) larvae. Aquaculture 257: 453-464.

45. Anagnostidis A, Michailidou M, Vatsos IN, Tsopelakos A, Miliou H, et al. (2015) Use of frozen mussel (Mytilus galloprovincialis) and mussel meal in the diet of sea bass (Dicentrarchus labrax Linnaeus, 1758) and sea bream (Sparus aurata Linnaeus, 1758) fingerlings - a preliminary study. Aquacult Res 46: 252-256.

46. Kroeckel S, Harjes AGE, Roth I, Katz H, Wuertz S, et al. (2012) When a turbot catches a fly: Evaluation of pre- pupae meal of the Black Soldier Fly (Hermetia illucens) as fish meal substitute - Growth performance and chitin degradation in juvenile turbot (Psetta maxima). Aquaculture 264-365: 345-352.

47. Cacerez-Martinez C (1984) Etudes sur les besoins nutritionnels de la sole (Solea vulgaris) et du turbot (Psetta maxima). Thése de Doctorat, Université de Bretagne Occidentale, Brest, France.

48. Leknes E, Imsland AK, Gústavsson A, Gunnarsson S, Thorarensen H, et al. (2012) Optimum feed formulation for turbot, Scophthalmus maximus (Rafinesque, 1810) in the grow-out phase. Aquaculture 344-349: 114-119.

49. Bonaldo A, Parma L, Mandrioli L, Sirri R, Fontanillas R, et al. (2011) Increasing dietary plant proteins affects growth performance and ammonia excretion but not digestibility and gut histology in turbot (Psetta maxima) juveniles. Aquaculture 318: 101-108.

50. Fournier V, Huelvan C, Desbruyeres E (2004) Incorporation of a mixture of plant feedstuffs as substitute for fish meal in diets of juvenile turbot (Psetta maxima). Aquaculture 236: 451-465.

51. Han QW, Liang MQ, Yao HB, Chang Q, Wu LX (2011) Effect of seven feed ingredients on growth performance, and liver and intestine histology of Lateolabrax japonicas. Prog Fish Sci 1: 32-39.

52. Hu L, Yun B, Xue M, Wang J, Wu X, et al. (2013) Effects of fish meal quality and fish meal substitution by animal protein blend on growth performance, flesh and liver histology of Japanese seabass. Aquaculture 372-375: 52-61.

53. Gatta PP, Parma L, Guarniero I, Mandrioli L, Sirri R, et al. (2011) Growth, feed utilization and liver histology of juvenile common sole (Solea solea L.) fed isoenergetic diets with increasing protein levels. Aquacult Res 42: 313-321.
This article was originally published in a special issue, Current and Emerging Diseases/Disorders of Fish in Aquaculture handled by Editor(s). Prof Patrick T.K. Woo, University of Guelph, Canada; Dr. Kenneth D.

Cain, University of Idaho, USA. 